\title{
Distinct Respiration and Physiological Changes during Flower Development and Senescence in Two Freesia Cultivars
}

\author{
Zhen Shu, Yimin Shi, Hongmei Qian, Yiwei Tao, and Dongqin Tang ${ }^{1}$ \\ School of Agriculture and Biology, Shanghai Jiao Tong University, 800 \\ Dongchuan Road, Shanghai 200240, China
}

Additional index words. Freesia hybrida, tepal, membrane lipid peroxidation, antioxidant enzyme, soluble compounds

\begin{abstract}
Two cultivars of Freesia hybrida, 'Shangnong Jinhuanghou' and 'Shangnong Hongtaige', were used to study the respiration rate and physiological responses during flower development and senescence. Phenotypically, the vase life of 'Shangnong Hongtaige' was significantly shorter than that of 'Shangnong Jinhuanghou'. At the whole flower level, both cultivars displayed similar change patterns on respiration rate. However, the change patterns in tepals, stamens, and pistils showed some differences in the two cultivars. A respiratory climacteric existed in most organs in both cultivars except for the stamen of 'Shangnong Jinhuanghou'. During flower development and senescence, the levels of soluble proteins and soluble sugars were very high at early stages, followed by a dramatic decrease, and the lowest levels occurred in wilted tepals in both cultivars. Superoxide dismutase (SOD) activities increased slightly at early developmental stages followed by a constant decrease in two cultivars, and SOD activities in 'Shangnong Jinhuanghou' were significantly higher than those in 'Shangnong Hongtaige'. Peroxidase activities showed a constant increase before tepals started wilting followed by a decrease in wilted tepals in both cultivars. In both cultivars, electrolytic leakage and malondialdehyde (MDA) content in tepals increased with the progression of development and senescence. MDA content in 'Shangnong Hongtaige' was much higher than that in 'Shangnong Jinhuanghou'. These results indicated that the respiratory climacteric, the decrease of antioxidant enzyme activities, the peroxidation of membrane lipid, and the loss of soluble compounds could be considered as indicators of flower senescence in Freesia.
\end{abstract}

Senescence is an age-dependent deterioration process at the cellular, tissue, organ, or organism level, leading to death or the end of the lifespan (Noodén, 1988). The wilting of flower petals is a deliberate and precisely controlled senescence event in plants and has attracted many studies on flower senescence. Studies suggest that much of the biochemistry of the petal cells' disassembly is shared and quite similar to the events that have been demonstrated in leaves and other organs (Reid and Chen, 2007). Senescence of plant tissues is generally accompanied by respiration increases; breakdown of carbohydrates, proteins, lipids, and nucleic acids (Singh et al., 2000); higher production of reactive oxygen species (ROS) (Del Rio et al., 1998); increased contents of malondialdehyde (MDA) (Hodges et al., 1999); and gradual loss in the ability of scavenging enzymes to neutralize free radicals (Kanazawa et al., 2000). It is necessary to elucidate the physiological mechanisms underlying the changes

Received for publication 29 Apr. 2010. Accepted for publication 23 May 2010.

The research was supported by the National Natural Science Foundation of China (No: 30872061).

${ }^{1}$ To whom reprint requests should be addressed; e-maildqtang@situ.edu.cn. and co-relationship of these factors to manipulate the flower senescence. To date, the importance of extending flower longevity to commercial floriculture has led to studies that have generated a wealth of information on physiological, biological, and molecular mechanisms of flower senescence in many floricultures such as petunia (Shibuya et al., 2004), rose (Kumar et al., 2008), and carnation (Wu et al., 1991).

Freesia is a kind of bulbous crops with high value for its colorful and aromatic flowers (Wang, 2007). Several studies on breeding, tissue culture, and postharvest biology of this crop had been performed (Qin and Lin, 1995; Wang, 2007). For instance, Spikman $(1986,1987,1989)$ found that Freesia flowers were sensitive to ethylene. van Meeteren et al. (1995) reported the relationships between carbohydrate and vase life of Freesia flowers. Very little is known, however, about the respiration characteristics and the physiological metabolisms during their flower development and senescence. The aim of our present study was to comparatively characterize the respiratory and physiological changes during flower development and senescence in Freesia hybrid and to provide physiological basis for its postharvest handling.
Plant materials and observation of vase life. Two Freesia cultivars (Qin and Lin, 1995), Freesia hybrida 'Shangnong Jinhuanghou' (which has single yellow flowers with normal stamens) and Freesia hybrida 'Shangnong Hongtaige' (which has double red flowers with abnormal stamens), were planted in the horticultural farm in Shanghai Jiao Tong University. Freesia spikes were harvested with stem length of $\approx 25 \mathrm{~cm}$ beneath the base of the inflorescence. After harvest, these spikes were kept in vessels filled with distilled water at room temperature and divided into five groups according to flower developmental stages. All buds or flowers used in the study were excised from the second basal (second) buds or flowers on the spikes.

Flower developmental stages were determined based on the second buds or flowers on the spikes. The phenotypes of different stages were described as follows: Stage 1, small, green bud $(\approx 1.5 \mathrm{~cm}$ in length); Stage 2 , bud at full color and ready to open; Stage 3, halfopen flower; Stage 4, fully open flower; and Stage 5, wilted flower.

Freesia spikes at Stage 2 were harvested and kept in vessels filled with distilled water at room temperature for observation of vase life. Individual flowers as well as whole inflorescences were observed. For the individual flower, the vase life of the second flower was considered started by Stage 2 and ended by Stage 5. For the whole inflorescence, the vase life was considered started when the first flower was ready to open and ended when the last flower was wilting and lost decorative value. Eight replicate spikes were used.

Measurement of respiration rate. For measurement of respiration rate, individual buds or flowers were excised from spikes and some were separated into different parts (tepals, stamens, and pistils) from the base of buds or flowers. For each stage (Stage 2 to Stage 5), one bud or flower, several tepals, several stamens, and several pistils were measured for their respiration rates at $25{ }^{\circ} \mathrm{C}$ using a Warburg respirometer method as described by Brown (1939) based on the $\mathrm{O}_{2}$ consumption. The respiration medium, Brodie solution $\left(1.033 \mathrm{~g} \cdot \mathrm{cm}^{-3}\right)$ that contained $46 \mathrm{~g} \mathrm{NaCl}, 10 \mathrm{~g}$ sodium cholate in $1 \mathrm{~L}$, was first injected into a manometer. Then $20 \%$ $\mathrm{KOH}$ solution was placed in the central cup in the vessel with the plant material, the top of the vessel was sealed, and then the whole unit was attached to the manometer for respiration measurement.

Quantification of soluble sugars and soluble proteins. One half gram of fresh tepal tissues was put into a tube with $10 \mathrm{~mL} 80 \%$ ethanol and the solution was collected (repeated twice), and then the solution was heated for $15 \mathrm{~min}$ in a boiling water bath. After cooling, the solution was centrifuged for $10 \mathrm{~min}$ at $3000 \mathrm{rpm}$ and the supernatant was collected to determine the contents of soluble sugars by using anthranone colorimetry with glucose as the standard (Hao et al., 2006). 
Tepal samples ( $0.5 \mathrm{~g}$ fresh weight) were homogenated with $5 \mathrm{~mL}$ distilled water (repeated twice). The mixture was collected and then centrifuged at $4000 \mathrm{rpm}$ for $10 \mathrm{~min}$ and the supernatant was collected to determine the contents of soluble proteins by the Coomassie Brilliant Blue G-250 method (Li, 2000) with bovine serum albumin as the standard.

Assay of antioxidant enzymes. Two grams of fresh tepal tissues were cut into pieces and homogenized in $100 \mathrm{~mm}$ phosphate buffer (pH 6.0) containing $1 \mathrm{~mm}$ EDTA- $\mathrm{Na}_{2}$ and $2 \%$ (w/v) PVP to extract peroxidase [POD, electrical conductivity (EC) 1.11.1.7]. Superoxide dismutase (SOD, EC 1.15.1.1) was extracted in $50 \mathrm{~mm}$ phosphate buffer ( $\mathrm{pH} 7.8$ ) by using $1 \mathrm{~g}$ of fresh tepal tissues. The extraction procedures were carried out on ice. The homogenate was centrifuged at $4000 \mathrm{rpm}$ for $15 \mathrm{~min}$ at $4{ }^{\circ} \mathrm{C}$ and the supernatant was collected for measuring the SOD activity.

SOD activity was spectrophotometrically assayed at $560 \mathrm{~nm}$ by the nitro-blue-tetrazolium (NBT) photoreduction method (Li, 2000). The reaction mixture $(3 \mathrm{~mL})$ contained $1.5 \mathrm{~mL}$ phosphate buffer (50 mM, pH 7.8), $2.0 \mu \mathrm{M}$ riboflavin, $13 \mathrm{~mm}$ methionine, $75 \mu \mathrm{M}$ NBT, 10 $\mu \mathrm{M}$ EDTA- $\mathrm{Na}_{2}$, and $100 \mu \mathrm{L}$ enzyme extracts. One unit of SOD activity was defined as the amount of extract that caused $50 \%$ inhibition in enzyme activity.

POD activity was determined at $25^{\circ} \mathrm{C}$ by using the guaiacol method (Guo, 2006) in a reaction mixture $(3 \mathrm{~mL})$ containing $20 \mathrm{~mm}$ guaiacol, $100 \mathrm{~mm}$ phosphate buffer $(\mathrm{pH} 7.0)$, $20 \mu \mathrm{L} 30 \%$ (w/v) $\mathrm{H}_{2} \mathrm{O}_{2}$, and $200 \mu \mathrm{L}$ enzyme extracts. The reaction was initiated by adding $\mathrm{H}_{2} \mathrm{O}_{2}$. One unit of POD activity was defined as the amount of enzyme required to increase 0.01 absorbance unit in the optical density at $470 \mathrm{~nm}$ per minute.

Determination of MDA content and electrolytic leakage. The MDA content was determined by the thiobarbituric acid method (Li, 2000). One half gram of fresh tepal tissues was ground in $5 \mathrm{~mL}$ trichloroacetic acid $(5 \%)$. After centrifuging at $3000 \mathrm{rpm}$ for $10 \mathrm{~min}$, the supernatant was kept at $4{ }^{\circ} \mathrm{C}$ for measuring the MDA concentration. Briefly, $2.5 \mathrm{~mL}$ of $5 \%$ trichloroacetic acid solution containing $0.5 \%$ thiobarbituric acid was added to a $1.5 \mathrm{~mL}$ aliquot of the supernatant. The mixture was heated in a boiling water bath for $15 \mathrm{~min}$ and then quickly cooled on ice. After centrifuging at $4500 \mathrm{rpm}$ for $10 \mathrm{~min}$, the supernatant was collected. Then the absorbance of the supernatant was read at $450 \mathrm{~nm}, 532 \mathrm{~nm}$, and $600 \mathrm{~nm}$ by a spectrophotometer. Distilled water was used as a control.

Electrolytic leakage (EL) values in tepals of different stages were measured as described by Hao et al. (2006). Twenty tepal discs (10 $\mathrm{mm}$ in diameter) were placed in a tube containing $30 \mathrm{~mL}$ deionized water and the initial value of $\mathrm{EC}$ was measured $\left(\mathrm{EC}_{0}\right)$ with a digital conductivity meter (Ecoscan con6, Klang Selangor D.E., Malaysia). The solution was kept at room temperature for $12 \mathrm{~h}$ and $\mathrm{EC}$ value was measured $\left(\mathrm{EC}_{1}\right)$ again. Then the solution was heated in a boiling water bath for $10 \mathrm{~min}$ and the final EC value was recorded
$\left(\mathrm{EC}_{2}\right)$ after cooling. The EL value was calculated using the following formula: $\mathrm{EL}(\%)=$ $\left[\left(\mathrm{EC}_{1}-\mathrm{EC}_{0}\right) /\left(\mathrm{EC}_{2}-\mathrm{EC}_{0}\right)\right] \times 100$.

Statistical analysis. All data presented were means of three replicates with SES. Statistical significance was determined by one-way analysis of variance using SPSS 11.5 for Windows (SPSS Inc., Chicago, IL). Differences between the means were assessed by Duncan's new multiple range test $(P<$ $0.05)$.

\section{Results}

The vase life of Freesia flower and changes in respiration rates. Phenotypically, the vase life of 'Shangnong Hongtaige' was shorter than that of 'Shangnong Jinhuanghou' $(P<0.05)$. The mean vase life of an individual flower was $6.4 \mathrm{~d}$ and $8.6 \mathrm{~d}$ for 'Shangnong Hongtaige' and 'Shangnong Jinhuanghou', respectively. The mean vase life of an inflorescence was $10.5 \mathrm{~d}$ and $14.1 \mathrm{~d}$ for

Table 1. Changes of the respiration rate $\left(\mathrm{O}_{2} \mu \mathrm{L} \cdot \mathrm{g}^{-1} /\right.$ fresh weight/h) in two Freesia cultivars during flower development and senescence.

\begin{tabular}{lccccc}
\hline Cultivar & Stage & Bud/flower & Tepal & Pistil & Stamen \\
\hline Shangnong & 2 & $30.80 \pm 1.79 \mathrm{~b}^{\mathrm{z}}$ & $33.75 \pm 0.58 \mathrm{c}$ & $59.49 \pm 1.17 \mathrm{~b}$ & $49.20 \pm 0.75 \mathrm{a}$ \\
Jinhuanghou & 3 & $35.24 \pm 1.24 \mathrm{a}$ & $36.61 \pm 0.56 \mathrm{~b}$ & $66.74 \pm 0.88 \mathrm{a}$ & $44.80 \pm 1.96 \mathrm{~b}$ \\
& 4 & $29.64 \pm 0.59 \mathrm{~b}$ & $42.24 \pm 0.95 \mathrm{a}$ & $38.18 \pm 1.09 \mathrm{~d}$ & $51.07 \pm 0.76 \mathrm{a}$ \\
& 5 & $24.29 \pm 0.98 \mathrm{c}$ & $31.53 \pm 1.00 \mathrm{c}$ & $48.39 \pm 1.17 \mathrm{c}$ & $15.45 \pm 1.17 \mathrm{c}$ \\
Shangnong & 2 & $33.16 \pm 1.03 \mathrm{~B}$ & $44.86 \pm 0.56 \mathrm{~A}$ & $67.03 \pm 0.44 \mathrm{~A}$ & ND $^{\mathrm{y}}$ \\
Hongtaige & 3 & $38.85 \pm 0.67 \mathrm{~A}$ & $35.32 \pm 1.11 \mathrm{~B}$ & $55.05 \pm 1.17 \mathrm{~B}$ & \\
& 4 & $35.56 \pm 0.08 \mathrm{~B}$ & $29.33 \pm 0.43 \mathrm{C}$ & $54.16 \pm 0.89 \mathrm{~B}$ & \\
& 5 & $26.88 \pm 0.70 \mathrm{C}$ & $37.46 \pm 0.43 \mathrm{~B}$ & $43.47 \pm 0.76 \mathrm{C}$ & \\
\hline
\end{tabular}

${ }^{2}$ Values in each column within the same cultivar followed by the same letter are not significantly different at $P<0.05$ based on Duncan's new multiple range test.

${ }^{y}$ Not measured because most stamens became small tepals.

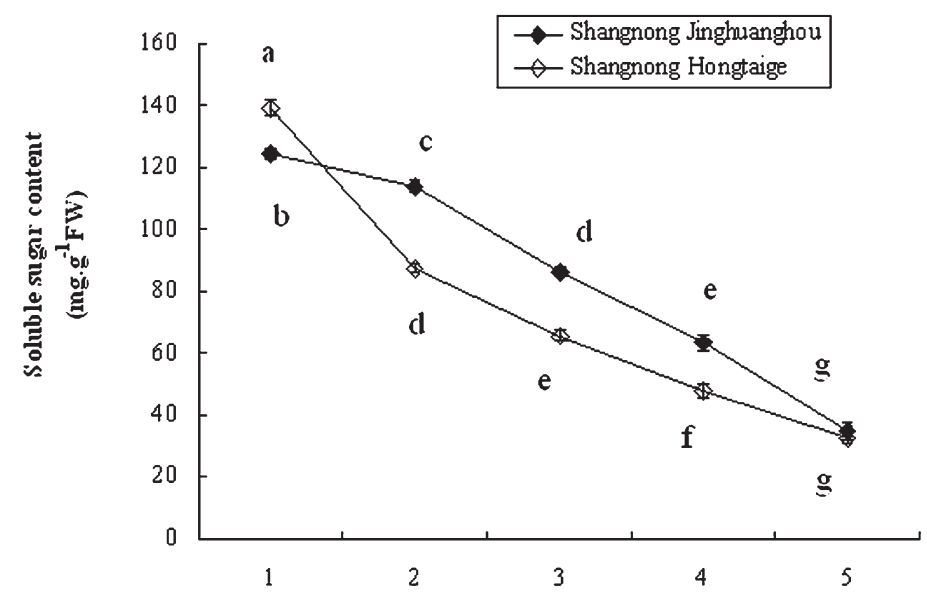

A

Stnge of flower developinent

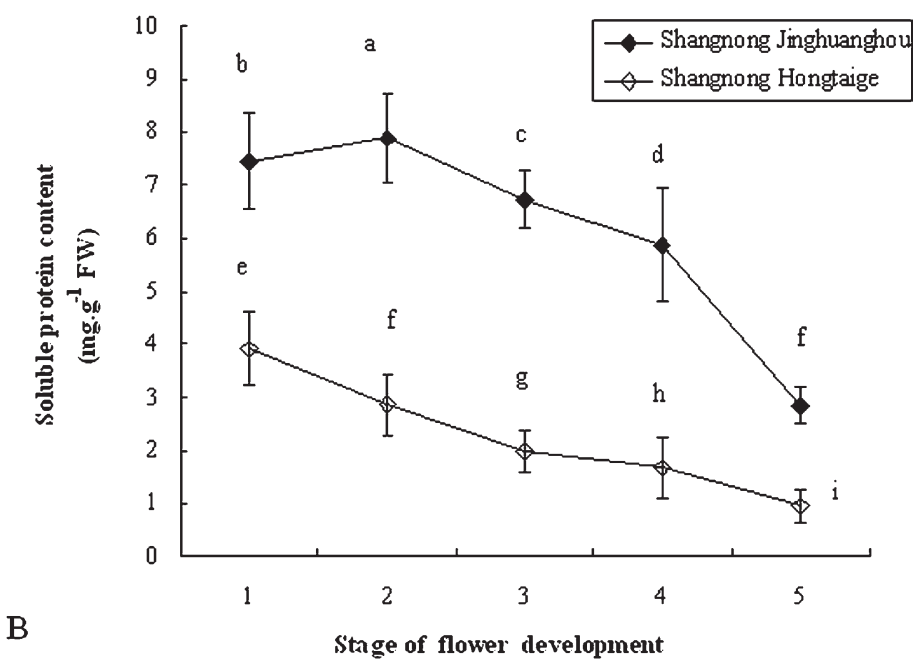

Fig. 1. Contents of soluble sugars (A) and soluble proteins (B) in two Freesia cultivars. Values followed by the same letter are not significantly different at $P<0.05$. 
'Shangnong Hongtaige' and 'Shangnong Jinhuanghou', respectively.

Similar change patterns in respiration rate were displayed at the whole flower level in both cultivars. As shown in Table 1, the respiration rate peaked after flower opening (Stage 3) and then declined with the senescence progression. The lowest was observed in flowers at Stage 5 , which was $\approx 30 \%$ of the highest. Although the overall change patterns were similar to each other, there were significant differences among young buds, open flowers, and wilted flowers in the two cultivars. Meanwhile, the change patterns of respiration rate in tepals, stamens, and pistils also showed some differences in both cultivars (Table 1). In 'Shangnong Jinhuanghou', a respiration climacteric was observed at Stage 4 in tepals; whereas in pistils, the highest respiration rate occurred at Stage 3 , and a dramatic decrease was observed at Stage 4 followed by a slight increase at Stage 5. In contrast, two respiration climacterics appeared at Stage 2 and Stage 4 in stamens, and a 70\% decrease was observed at Stage 5, which was different from the change pattern in, petals, pistils, and whole flowers. In 'Shangnong Hongtaige', the highest respiration rates were observed at Stage 2 in both tepals and pistils, which were earlier than that in 'Shangnong Jinhuanghou'. Because stamens in 'Shangnong Hongtaige' became small tepals, their respiration rate was not measured in the present study.

Changes in contents of soluble sugars and soluble proteins. In both cultivars, the highest levels of soluble sugars were observed at Stage 1 followed by a rapid decrease with the senescence progression, whereas the lowest levels of soluble sugars occurred at Stage 5 , which were only $28 \%$ and $23 \%$ of the highest levels in 'Shangnong Jinhuanghou' and 'Shangnong Hongtaige', respectively (Fig. 1A). The results also showed that a more rapid decrease occurred in 'Shangnong Hongtaige' than that in 'Shangnong Jinhuanghou' from Stage 1 to Stage 4.

During flower development and senescence, the contents of soluble proteins showed a decrease trend in both cultivars except a slight increase at Stage 2 in 'Shangnong Jinhuanghou' (Fig. 1B). The lowest, only 36\% and $24 \%$ of the highest, was observed at Stage 5 in 'Shangnong Jinhuanghou' and 'Shangnong Hongtaige', respectively. A significant difference $(P<0.05)$ was also shown in contents of soluble proteins in two cultivars. In 'Shangnong Jinhuanghou', the protein levels in petals of each stage were two to three times higher than those in 'Shangnong Hongtaige'.

Activities of some antioxidant enzymes. SOD activities in tepals showed a continuous decrease with the senescence progression in both cultivars (Fig. 2A). The highest SOD activities were 2.4 and 1.8 times the lowest values (at Stage 5) in 'Shangnong Jinhuanghou' and 'Shangnong Hongtaige', respectively. Meanwhile, SOD activities in 'Shangnong Jinhuanghou' were higher than those in 'Shangnong Hongtaige' $(P<0.05) ; 1.5$ to 2.8 times of SOD activities were observed in 'Shangnong Jinhuanghou' than those in 'Shangnong Hongtaige'.
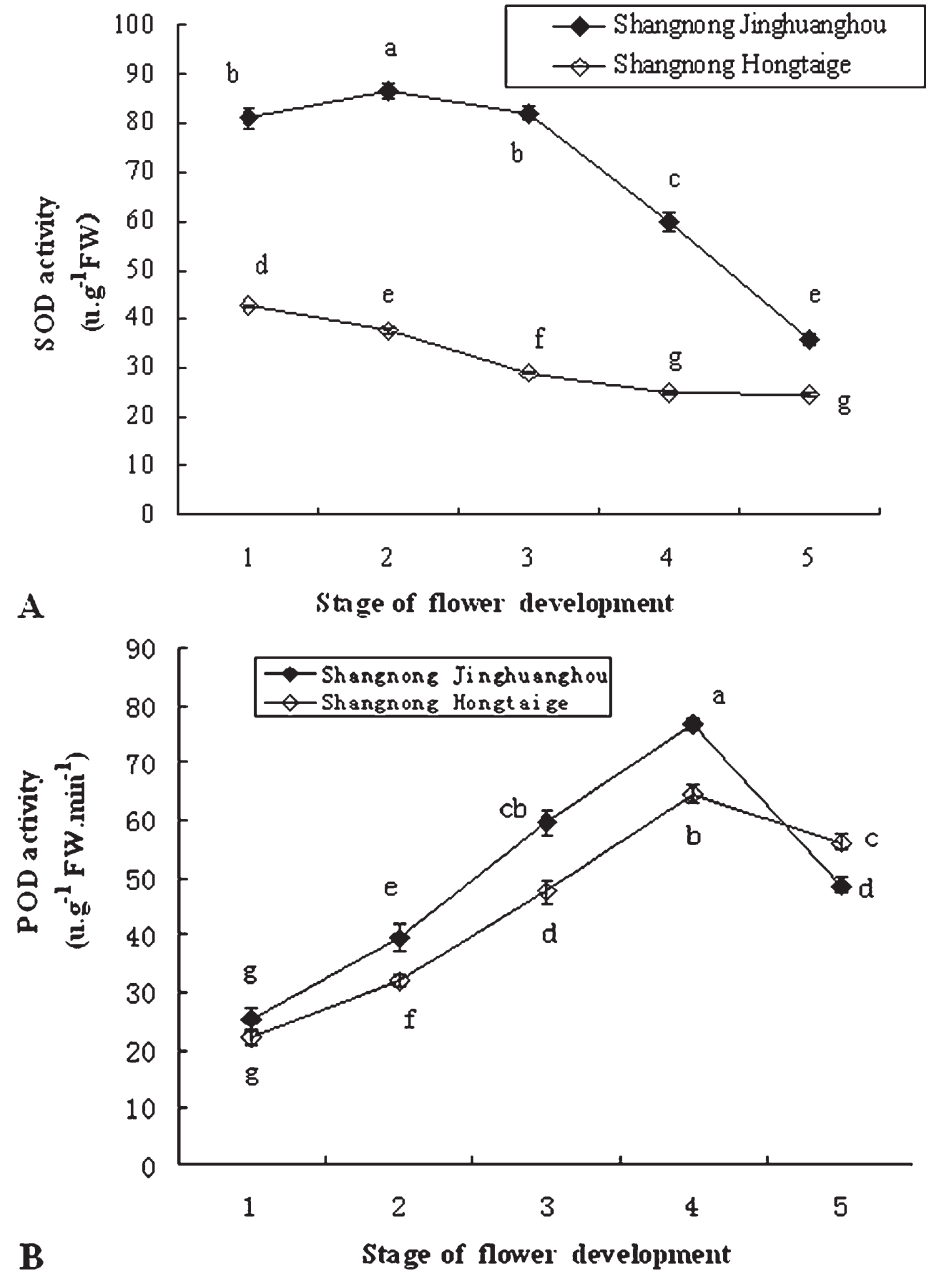

Fig. 2. Activities of superoxide dismutase (SOD) (A) and peroxidase (POD) (B) in two Freesia cultivars. Values followed by the same letter are not significantly different at $P<0.05$.

In contrast, POD activities increased gradually during early four stages and reached the highest at Stage 4 and then decreased at Stage 5 in both cultivars (Fig. 2B). Approximately three-time increases of POD activities were observed from Stage 1 to Stage 4 in both cultivars. There were also higher POD activities in 'Shangnong Jinhuanghou' than those in 'Shangnong Hongtaige' from Stage 1 to Stage 4.

Lipid peroxidation and membrane permeability. MDA content in the two cultivars showed a significant $(P<0.05)$ increase during flower development and senescence (Fig. 3A). In 'Shangnong Jinhuanghou', the highest was observed at stage 5 [330.3 $\mathrm{nmol} \cdot \mathrm{g}^{-1}$ fresh weight $\left.(\mathrm{FW})\right]$, which was much higher than the lowest at stage 1 (72.3 $\left.\mathrm{nmol} \cdot \mathrm{g}^{-1} \mathrm{FW}\right)$. The same change pattern was observed in 'Shangnong Hongtaige'. However, MDA contents in this cultivar were significantly higher than those in 'Shangnong Jinhuanghou' $(P<0.05)$, especially at early developmental stages. The difference of MDA contents in the two cultivars became less with senescence progression.

The membrane permeability was evaluated by electrolytic leakage. As shown in Figure 3B, EL increased significantly $(P<$
$0.05)$ with the senescence progression and the highest was observed at Stage 5. In both cultivars, an approximately four times increase of EL was observed from Stage 1 to Stage 4.

\section{Discussion}

The objective of our experiments was to analyze the physiological mechanisms of flower development and senescence in Freesia.

Respiration is a major source of ROS (Rich and Bonner, 1978). The respiratory climacteric, which existed in both Freesia cultivars, was probably a significant contributing factor to the increasing oxidative stress with the senescence progression. As a result of the increase in respiration, the production of ROS probably increases after flower opening in Freesia. In both cultivars, the respiration rate in pistils was higher than that in tepals and stamens at most stages, suggesting that the respiration of pistils plays a more important role than those of other tissues in Freesia flower senescence.

Carbohydrates are primary energy source in plants (Gibson, 2004); some are consumed as respiratory substrates and some others are converted to other compounds. Changing 

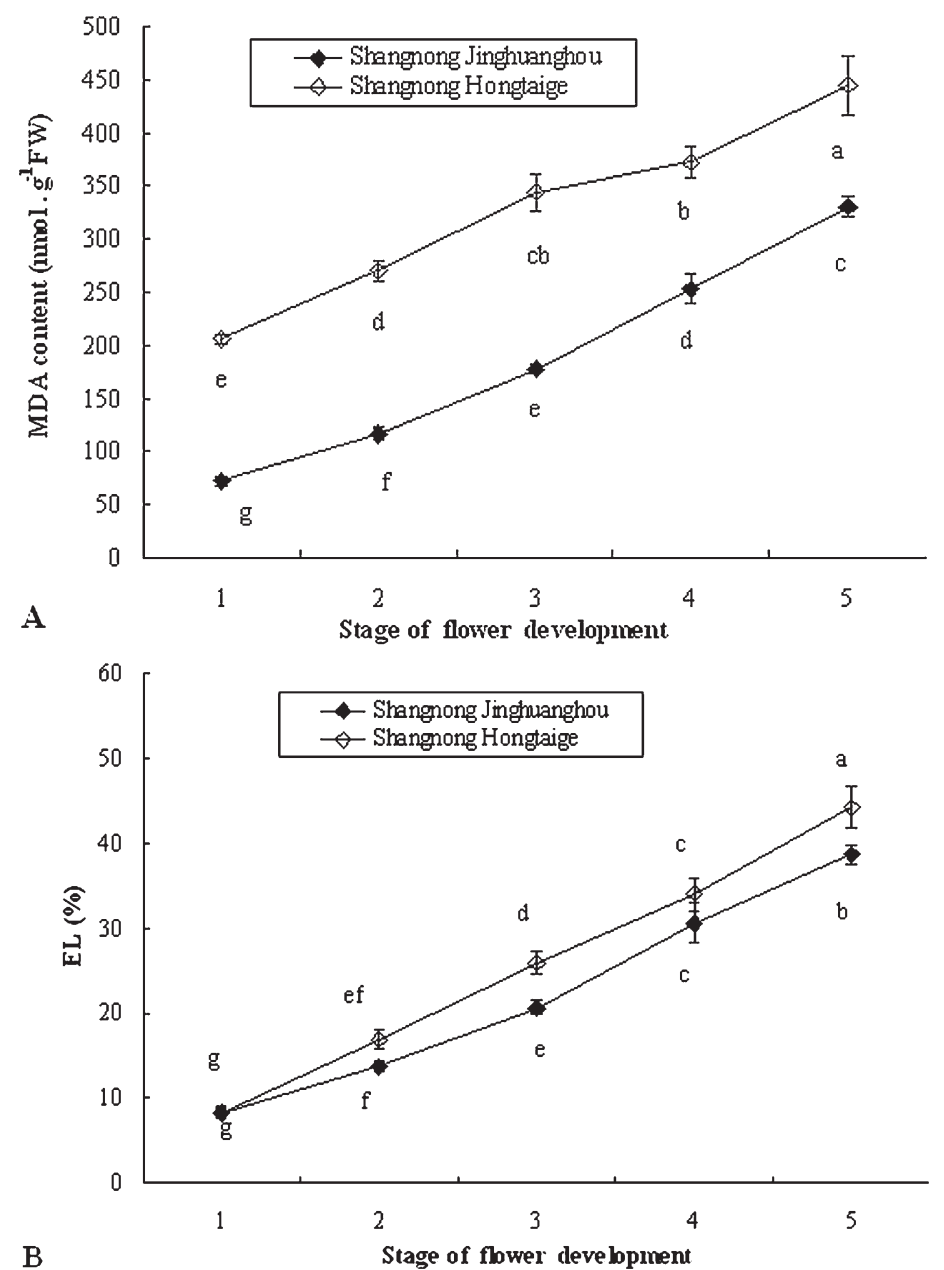

Fig. 3. malondialdehyde (MDA) content (A) and electrolytic leakage (EL) (B) in two Freesia cultivars. Values followed by the same letter are not significantly different at $P<0.05$.

source-sink relations are a common event during plant development (Roitsch and Gonzalez, 2004). Flower senescence also allows for nutrient recycling (Reid and Chen, 2007). In our study, a dramatic decrease of soluble sugars in Freesia flowers was observed, revealing that sugar starvation might be a cause of tepal senescence in Freesia. Meanwhile, a more rapid decrease of soluble sugars occurred in 'Shangnong Hongtaige', revealing earlier sugar starvation occurred in this cultivar.

The protein content has a close link with the flower senescence (Borochov and Woodson, 1989). The decrease of protein content was one of the important indices of the senescence in plant flowers (Halevy and Mayak, 1981; Song, 1998) and protein degradation is an important part of the remobilization process during flower senescence (Reid and Chen, 2007). A decrease in overall protein levels can result from a decrease in synthesis, an increase in degradation, or both (Stephenson and Rubinstein, 1998; van Doorn and Woltering, 2008). The results showed that the decrease of soluble proteins in Freesia tepals occurred before visible senescence symptoms, revealing the protein degradation increased with the senescence progression. The lower levels of soluble proteins in 'Shangnong Hongtaige' suggested a lower capacity of the protein synthesis (including antioxidative enzymes).

SOD converts superoxide $\left(\mathrm{O}_{2}{ }^{-}\right)$into molecular oxygen $\left(\mathrm{O}_{2}\right)$ and hydrogen peroxide $\left(\mathrm{H}_{2} \mathrm{O}_{2}\right)$, and POD is implicated in the scavenging of $\mathrm{H}_{2} \mathrm{O}_{2}$ (Møller et al., 2007; Scandalios, 1993). High SOD activities at early stages in Freesia tepals mean high efficiency in the reduction of $\mathrm{O}_{2}^{-}$. The low SOD efficiency in tepals at later stages led to the lipid peroxidation and the accumulation of $\mathrm{O}_{2}{ }^{-}$ that further accelerated the senescence process. This was consistent with the increased rate of lipid peroxidation represented by MDA content. On the other hand, the increase of POD activities the four early stages indicated a continuously enhanced capability of reducing $\mathrm{H}_{2} \mathrm{O}_{2}$. The oxidation reaction may be promoted by the $\mathrm{H}_{2} \mathrm{O}_{2}$ from the reduction of $\mathrm{O}_{2}{ }^{-}$. Decrease of POD activity in wilted tepals, which was probably the result of the increase of protein degradation, revealed that deleterious effects of ROS were exceeding the ability to counteract the cytotoxic compounds. The difference in initial time for SOD and POD activity to reach the highest in Freesia tepals suggested the possibility to regulate the formation of $\mathrm{O}_{2}{ }^{-}$during senescence. In addition, lower activities of
SOD and POD in 'Shangnong Hongtaige' also suggested that the reduction of ROS scavenging capacity accelerated the senescing process in this cultivar.

The changes in SOD and POD activities broke the balance between the generation and neutralization of ROS. The accumulation of ROS resulted in the peroxidation of membrane lipid and further affected the membrane permeability. The significant increase of MDA indicated that the lipid peroxidation caused by ROS occurred with the senescence progression in Freesia flowers. In addition, the higher MDA content in 'Shangnong Hongtaige' suggested that its membrane deterioration was much more severe than that in 'Shangnong Jinhuanghou'. During petal senescence, cellular membranes lose their integrity progressively, resulting in leakage of pigments, nutrients, and electrolytes from the cells (Rubinstein, 2000). A significant increase in EL was also shown with the senescence progression in Freesia flowers, revealing the damage of membrane occurred before the visible senescence phenotype, which resulted in endocyte loss and further accelerated the senescence process in Freesia flowers. In general, the accumulation of MDA was accompanied by the increase of EL. However, much greater increase in MDA content was observed than the EL in the two cultivars. In 'Shangnong Hongtaige', other mechanisms should be triggered to protect the membrane stability from the damage by severer lipid peroxidation. Further study to elucidate this special phenomenon of MDA and EL change in Freesia should be interesting.

The vase life of 'Shangnong Hongtaige' was significantly shorter than that of 'Shangnong Jinhuanghou'. The earlier high respiration rate in tepals and pistils, faster decrease of soluble sugar content, lower protein content level, lower activities of SOD and POD, and higher MDA content in 'Shangnong Hongtaige' might be the causes for its shorter vase life compared with the other cultivar.

In conclusion, the results suggested that the balance of physiological metabolism was broken with the senescence progression in Freesia flowers. Respiratory climacteric, rapid loss of soluble compounds, decrease of antioxidative enzymes activities, increases of MDA content, and EL could be considered the indicators of flower senescence in Freesia.

\section{Literature Cited}

Borochov, A. and W.R. Woodson. 1989. Physiology and biochemistry of flower petal senescence. Hort. Rev. (Amer. Soc. Hort. Sci.) 11:15-43.

Brown, J.W. 1939. Suggestions for the use of Warburg respirometers in plant physiological investigations. Plant Physiol. 14:309-320.

Del Rio, L.A., G.M. Pastori, J.M. Palma, L.M. Sandalio, F. Sevilla, F.J. Corpas, A. Jimenez, F. Lopez-Huertas, and J.A. Hernandez. 1998. The activated oxygen role of peroxisomes in senescence. Plant Physiol. 116:1195-1200.

Gibson, S.I. 2004. Sugar and phytohormone response pathways: Navigating a signaling network. J. Expt. Bot. 55:253-264. 
Guo, J.F. (ed.). 2006. Laboratory manual of plant physiology. Higher Education Press, Beijing, China [in Chinese].

Halevy, A.H. and S. Mayak. 1981. Senescence and postharvest physiology of cut flowers. Part 2. Hortic. Rev. (Am. Soc. Hortic. Sci.) 3:59-143.

Hao, J.J., Z.L. Kang, and Y. Yu (eds.). 2006. Experimental technique of plant physiology. Chemical Industry Press, Beijing, China [in Chinese].

Hodges, D.M., J.M. DeLong, C. Forney, and R.K. Prange. 1999. Improving the thiobarbituric acid reactive-substances assay for estimating lipid peroxidation in plant tissues containing anthocyanin and other interfering compounds. Planta 207:604-611.

Kanazawa, S., S. Savo, T. Koshiba, and S. Sano. 2000. Changes in antioxidative enzymes in cucumber cotyledons during natural senescence: Comparison with those during dark induced senescence. Physiol. Plant. 109:211-216.

Kumar, N., G.C. Srivastava, and K. Dixit. 2008. Flower bud opening and senescence in roses (Rosa hybrida L.). Plant Growth Regulat. 55: 81-99.

Li, H.S. (ed.). 2000. Experimental principles and technique of plant physiology and biochemistry. Higher Education Press, Beijing, China [in Chinese].

Møller, I.M., P.E. Jensen, and A. Hansson. 2007. Oxidative modifications to cellular components in plants. Annu. Rev. Plant Biol. 58: 459-481.
Noodén, L.D. 1988. The phenomena of senescence and aging, p. 1-50. In: Noodén, L.D. and A.C. Leopold (eds.). Senescence and aging in plants. Academic Press in London, San Diego, CA.

Qin, W.Y. and Y.X. Lin (eds.). 1995. Research on Freesia. Shanghai Science and Technology Press, Shanghai, China. [in Chinese].

Reid, M.S. and J.C. Chen. 2007. Flower senescence, p. 256-277. In: Gan, S.S. (ed.). Senescence processes in plants.. Blackwell Publishing, Oxford, UK.

Rich, P. and W.D. Bonner. 1978. The sites of superoxide anion generation in higher plant mitochondria. Arch. Biochem. Biophys. 188: 206-213.

Roitsch, T. and M.C. Gonzalez. 2004. Function and regulation of plant invertases: Sweet sensations. Trends Plant Sci. 9:606-613.

Rubinstein, B. 2000. Regulation of cell death in flower petals. Plant Mol. Biol. 44:303-318.

Scandalios, J.G. 1993. Oxygen stress and superoxides dismutase. Plant Physiol. 101:7-12.

Shibuya, K., K.G. Barry, J.A. Ciardi, H.M. Loucas, B.A. Underwood, S. Nourizadeh, J.R. Ecker, H.J. Klee, and D.G. Clark. 2004. The central role of PhEIN2 in ethylene responses throughout plant development in petunia. Plant Physiol. 136:2900-2912.

Singh, M., K. Dhawan, S.P. Malhotra, and R. Singh. 2000. Carbohydrate metabolism in tomato (Lycopersicon esculentum L. Mill) fruits during ripening. J. Food Sci. Technol $37: 222-226$
Song, C.P. (ed.). 1998. Plant senescence biology. Beijing University Press, Beijing, China [in Chinese].

Spikman, G. 1986. The effect of water stress on ethylene production and ethylene sensitivity of freesia inflorescences. Acta Hort. 181:134 140 .

Spikman, G. 1987. Ethylene production, ACC and MACC content of freesia buds and florets. Sci. Hort. 33:291-297.

Spikman, G. 1989. Development and ethylene production of buds and florets of cut freesia inflorescences as influenced by silver thiosulphate, aminoethoxyvinylglycine and sucrose. Sci. Hort. 39:73-81.

Stephenson, P. and B. Rubinstein. 1998. Characterization of proteolytic activity during senescence in daylilies. Physiol. Plant. 104:463-473.

van Doorn, W.G. and E.J. Woltering. 2008. Physiology and molecular biology of petal senescence. J. Expt. Bot. 59:453-480

van Meeteren, U., H. van Gelder, and A.C. van de Peppel. 1995. Aspects of carbohydrate balance during floret opening in freesia. Acta Hort. 405:117-122.

Wang, L. 2007. Freesia, p. 665-693. In: Anderson, N.O. (ed.). Flower breeding and genetics. Springer, New York.

Wu, M.J., W.G. van Doorn, and M.S. Reid. 1991 Variation in the senescence of carnation ( $\mathrm{Di}$ anthus caryophyllus L.) cultivars. I. Comparison of flower life, respiration and ethylene biosynthesis. Sci. Hort. 48:99-108. 